\title{
ASYMPTOTIC METHODS OF ENUMERATION AND APPLICATIONS TO MARKOV CHAIN MODELS
}

\author{
MICHAEL DRMOTA
}

\begin{abstract}
The purpose of this article is to present analytic methods for determining the asymptotic behaviour of the coefficents of power series that can be applied to homogeneous discrete quasi death and birth processes. It turns that there are in principle only three types for the asymptotic behaviour. The process either converges to the stationary distribution or it can be approximated in terms of a reflected Brownian motion or by a Brownian motion. In terms of Markov chains these cases correspond to positive recurrence, to null recurrence, and to non recurrence. The same results hold for the continuous case, too.
\end{abstract}

\section{INTRODUCTION}

Let

$$
y(x)=\sum_{n \geq 0} y_{n} x^{n}
$$

be a power series (or generating function) of non-negative real numbers $y_{n} \geq 0$ that represents an analytic function for $|x|<R$, where

$$
R=\left(\limsup _{n \rightarrow \infty} y_{n}^{1 / n}\right)^{-1}
$$

is the radius of convergence. It is well known that $y(x)$ is singular at $x_{0}=R$ and thus the smallest singularity (or equivalently the radius of convergence) provides a first infomation on the asympotic behaviour of $y_{n}$. In particular, for every $\varepsilon>0$ there exists $C_{1}>0$ and $C_{2}>0$ such that

$$
y_{n} \leq C_{1} R^{-n}(1+\varepsilon)^{n}
$$

for all $n \geq 0$ and

$$
y_{n} \geq C_{2} R^{-n}(1-\varepsilon)^{n}
$$

for infinitely many $n \geq 0$. Furthermore, if one knows the behaviour of $y(x)$ for $x \rightarrow R$ - one can be even a little bit more precise. Since $y_{n} r^{n} \leq y(r)$ for $0<r<R$ one also gets

$$
y_{n} \leq \min _{0<r<R} y(r) r^{-n} .
$$

Already these simple observations indicate that the knowledge of the analytic behaviour of the complex function $y(x)$, that is, the location of the singularties and the local behaviour of $y(x)$ around them, provides information on the asymptotic

Date: December 31, 2004.

Institut für Diskrete Mathematik und Geometrie, TU Wien, Wiedner Hauptstrasse 8-10/104, A-1040 Wien, Austria, email: michael.drmota@tuwien.ac.at. 
behaviour of the coefficients $y_{n}$. Of course, if one knows $y(x)$ exactly then $y_{n}$ can be recovered by Cauchy's formula ${ }^{1}$

$$
y_{n}=\left[x^{n}\right] y(x)=\frac{1}{2 \pi i} \int_{|x|=r} y(x) x^{-n-1} d x
$$

Thus, the main problem in this context is to observe how much information on $y(x)$ is needed to expand this contour integral as precisely as possible.

In what follows (see section 2) we will make this statement more clearly. We will collect some known facts on transfer principles (singularity analysis, saddle point method), where the kind of singularity of $y(x)$ resp. precise growth properties of $y(x)$ reflect the asymptotic behaviour of the coefficients $y_{n}$.

These kinds of methods are widely applied to asymptotic enumeration problems (see $[1,12])$. In this context the coeffients $y_{n}$ are certain (combinatorial) numbers of interest (e.g. number of combinations, partitions, permutations etc. with some properties), where the recursive structure of the combinatorial problem can be translated into proper explicit or implicit relations for the corresponding generating functions, compare with [15]. There are also many applications in the combinatorial analysis of data structures and algorithms, see [14].

In this survey we will apply this asymptotic enumeration procedure to some special Markov processes $X_{n}$, namely to homogeneous quasi birth and death processes (see $[10,11,9])$. This processes can be viewed as random walks on properly defined "periodic" infinite graphs and also, the probabilities of the distribution of the random walk can be interpreted as a weighted counting problem for paths on that graph. With help of this combinatorial interpretation we derive (more or less) explicit representations for the generating functions of the corresponding probabilities. At this stage we can apply proper transfer principles to obtain asymptotic representations for the probabilites in question.

It turns out that there are three typical cases, the positive recurrent one, the null recurrent one and the non-recurrent one. In the first case $X_{n}$ has a discrete limiting distribution, in the second one $X_{n}$ can be approximated im terms of a reflected Brownian motion, and in the third case in terms of a Brownian motion. In the continuous case we get (in principle) the same results but we have to use the Laplace transform instead of generating function. These results are only partly new, e.g. the postive recurrent case is very well studied, see [9]. However, it seems that this approach is novel for these kinds of problems. The advantage of this approach is that one gets very precise information on the distribution of $X_{n}$. The discrete case (Theorems 1 and 2) has been already discussed by the author [5] in terms of random walks on graphs, the continuous case is new in this context.

The structure of the paper is the following one. In Section 2 we collect some results (with proofs) on asymptotic expansions for coefficients of generating functions. In Section 3 we present a combinatorial approach to homogeneous quasi birth and death processes (QBD's) with help of generating functions (and Laplace transforms). Finally, in Section 4 we apply the methods of section 2 to these QBD's to obtain the above mentioned limiting relations for $X_{n}$.

${ }^{1}$ We will always use the notation $\left[x^{n}\right] y(x)=y_{n}$ to denote the $n$-th coefficient of a power series. 


\section{Analytic Methods}

2.1. Singularity Analysis. We start with the analysis of algebraic singularities. There exists very general transfer principles that are due to Flajolet and Odlyzko [6] and can be considered as analytic Tauberian theorems. The main difference to (real) Tauberian theorems is that one assumes that the analytic function $y(x)$ can be analytically continued to a region $\Delta$ that is a little bit larger than the circle of convergence. A combination of Lemma 1 and 2 is then a very powerful tool to obtain asymptotic expansions of the coefficients of analytic functions with effective error terms in an almost automatic way.

Lemma 1. Let

$$
y(x)=\sum_{n \geq 0} y_{n} x^{n}
$$

be analytic in a region

$$
\Delta=\left\{x:|x|<x_{0}+\eta,\left|\arg \left(x-x_{0}\right)\right|>\delta\right\},
$$

in which $x_{0}$ and $\eta$ are positive real numbers and $0<\delta<\pi / 2$. Furthermore suppose that there exists a real number $\alpha$ such that

$$
y(x)=\mathcal{O}\left(\left(1-x / x_{0}\right)^{-\alpha}\right) \quad(x \in \Delta) .
$$

Then

$$
y_{n}=\mathcal{O}\left(x_{0}^{-n} n^{\alpha-1}\right) .
$$

Proof. One uses Cauchy's formula

$$
y_{n}=\frac{1}{2 \pi i} \int_{\gamma} y(x) x^{-n-1} d x
$$

where $\gamma$ is a suitable chosen path of integration around the origin. In particular one can use $\gamma=\gamma_{1} \cup \gamma_{2} \cup \gamma_{3} \cup \gamma_{4}$, where

$$
\begin{aligned}
& \gamma_{1}=\left\{x=x_{0}+\frac{z}{n}:|z|=1, \delta \leq|\arg (z)| \leq \pi\right\}, \\
& \gamma_{2}=\left\{x=x_{0}+t e^{i \delta}: \frac{1}{n} \leq t \leq \eta\right\}, \\
& \gamma_{3}=\left\{x=x_{0}+t e^{-i \delta}: \frac{1}{n} \leq t \leq \eta\right\}, \\
& \gamma_{4}=\left\{x:|x|=\left|x_{0}+e^{i \delta} \eta\right|, \arg \left(x_{0}+e^{i \delta} \eta\right) \leq|\arg x| \leq \pi\right\} .
\end{aligned}
$$

It is easy to show that the bound $|y(z)| \leq C\left|1-z / x_{0}\right|^{-\alpha}$ directly proves that

$$
\left|\int_{\gamma_{1} \cup \gamma_{2} \cup \gamma_{3}} \frac{y(z)}{z^{n+1}} d z\right|=\mathcal{O}\left(x_{0}^{-n} n^{\alpha-1}\right),
$$

whereas the integal over $\gamma_{4}$ is exponentially smaller: $\mathcal{O}\left(\left(x_{0}+\eta\right)^{-n}\right)$.

Remark . Note that the same proof method also shows that

$$
y(x)=o\left(\left(1-x / x_{0}\right)^{-\alpha}\right) \quad(x \in \Delta) .
$$

implies

$$
y_{n}=o\left(x_{0}^{-n} n^{\alpha-1}\right)
$$

as $n \rightarrow \infty$. 
Furthermore, it would have been sufficient to use a variating $\eta=\eta(n)$ of the form $\eta=(\log n)^{2} / n$. Then the integral over $\gamma_{4}$ is not exponentially smaller but of order $\mathcal{O}\left(\left(x_{0}\right)^{-n} e^{-c(\log n)^{2}}\right)$ which is better than any polynomial decay.

Lemma 1 is complemented by the following well known property on the asymptotic expansion of binomial coefficients. We include a proof since the same contour integration (that is also very close to the proof of Lemma 1) will used in section 2.3 , too.

Lemma 2. Suppose that $y(x)=(1-x)^{-\alpha}$. Then

$$
y_{n}=(-1)^{n}\left(\begin{array}{c}
-\alpha \\
n
\end{array}\right)=\frac{n^{\alpha-1}}{\Gamma(\alpha)}+\mathcal{O}\left(n^{\alpha-2}\right) .
$$

Proof. We again use Cauchy's formula for the contour $\gamma=\gamma_{1} \cup \gamma_{2} \cup \gamma_{3} \cup \gamma_{4}$, where

$$
\begin{aligned}
& \gamma_{1}=\left\{x=1+\frac{t}{n}|| t \mid=1, \Re t \leq 0\right\} \\
& \gamma_{2}=\left\{x=1+\frac{t}{n} \mid 0<\Re t \leq \log ^{2} n, \Im t=1\right\} \\
& \gamma_{3}=\frac{1}{\gamma_{2}} \\
& \gamma_{4}=\left\{x|| x|=| 1+\frac{\log ^{2} n+i}{n}\left|, \arg \left(1+\frac{\log ^{2} n+i}{n}\right) \leq\right| \arg (x) \mid \leq \pi\right\} .
\end{aligned}
$$

As above, the integral over $\gamma_{4}$ is negligible.

For the remaining part we approximate $x^{-n-1}$ by $e^{-t}\left(1+\mathcal{O}\left(t^{2} / n\right)\right)$. Hence, the integral over $\gamma_{1} \cup \gamma_{2} \cup \gamma_{3}$ is given by

$$
\begin{aligned}
\frac{1}{2 \pi i} \int_{\gamma_{1} \cup \gamma_{2} \cup \gamma_{3}}(1-x)^{-\alpha} x^{-n-1} d x & =\frac{n^{\alpha-1}}{2 \pi i} \int_{\gamma^{\prime}}(-t)^{-\alpha} e^{-t} d t \\
& +\frac{n^{\alpha-2}}{2 \pi i} \int_{\gamma^{\prime}}(-t)^{-\alpha} e^{-t} \cdot \mathcal{O}\left(t^{2}\right) d t \\
& =n^{\alpha-1} I_{1}+\mathcal{O}\left(n^{\alpha-2}\right)
\end{aligned}
$$

where $\gamma^{\prime}=\{t|| t \mid=1, \Re t \leq 0\} \cup\left\{t \mid 0<\Re t \leq \log ^{2} n\right.$, ऽt $\left.= \pm 1\right\}$. Now $I_{1}$ approximates $1 / \Gamma(\alpha)$ (by Hankel's integral representation) in the following way:

$$
\begin{aligned}
I_{1} & =\frac{1}{\Gamma(\alpha)}+\mathcal{O}\left(\int_{(\log n)^{2}}^{\infty} e^{2 \pi|\alpha|}\left(1+t^{2}\right)^{-\frac{1}{2} \Re \alpha} e^{-t} d t\right) \\
& =\frac{1}{\Gamma(\alpha)}+\mathcal{O}\left(\left(1+(\log n)^{4}\right)^{-\frac{1}{2} \Re \alpha} e^{-(\log n)^{2}}\right)=\frac{1}{\Gamma(\alpha)}+\mathcal{O}\left(\frac{1}{n}\right) .
\end{aligned}
$$

Of course, this completes the proof of Lemma 2.

Remark . These two lemmas can be now used to transfer an asymptotic series expansion of $y(x)$ to an asymptotic series expansion for $y_{n}$. Suppose that a function $y(x)$ is analytic in a region of the form $\Delta$ and that it has an expansion of the form

$$
y(x)=C\left(1-\frac{x}{x_{0}}\right)^{-\alpha}+\mathcal{O}\left(\left(1-\frac{x}{x_{0}}\right)^{-\beta}\right) \quad(x \in \Delta),
$$


where $\beta<\alpha$. Then we have

$$
y_{n}=\left[x^{n}\right] y(x)=C \frac{n^{\alpha-1}}{\Gamma(\alpha)} x_{0}^{-n}+\mathcal{O}\left(x_{0}^{-n} n^{\max \{\alpha-2, \beta-1\}}\right) .
$$

Note that $(-1)^{n}\left(\begin{array}{c}-\alpha \\ n\end{array}\right)$ has a full asymptotic series expansion of the form

$$
(-1)^{n}\left(\begin{array}{c}
-\alpha \\
n
\end{array}\right) \sim \frac{n^{\alpha-1}}{\Gamma(\alpha)}\left(1+\sum_{k \geq 1} \frac{e_{k}(\alpha)}{n^{k}}\right),
$$

where

$$
e_{k}(\alpha)=\sum_{l=k}^{2 k} \lambda_{k, l}(\alpha-1)(\alpha-2) \cdots(\alpha-l)
$$

with

$$
\sum_{k, l \geq 0} \lambda_{k, l} v^{k} t^{l}=e^{t}(1+v t)^{-1-1 / v}
$$

This and some related formulae can be found in [6].

Finally we want to mention that polar singularities are even more easy to handle. (The proof is immediate.)

Lemma 3. Suppose that $y(x)$ is a meromorphic functions that is analytic at $x=0$ and has polar singularities at the points $q_{1}, \ldots, q_{r}$ in the circle $|x|<R$, that is, $y(x)$ has a representation of the form

$$
y(x)=\sum_{j=1}^{r} \sum_{k=1}^{\lambda_{j}} \frac{B_{j k}}{\left(1-x / q_{j}\right)^{k}}+T(x),
$$

where $T(x)$ is analytic in the region $|x|<R$. Then for every $\varepsilon>0$

$$
\left[x^{n}\right] y(x)=\sum_{j=1}^{r} \sum_{k=1}^{\lambda_{j}} B_{j k}\left(\begin{array}{c}
n+k-1 \\
k-1
\end{array}\right) q_{j}^{-n}+\mathcal{O}\left(R^{-n}(1+\varepsilon)^{n}\right) .
$$

2.2. Systems of Functional Equations. Next, we will show that squareroot singularities naturally appear in a system of functional equations. Lemma 4 will a also crucial for the proof of the null recurrenct case of Theorem 2. Our presentation is very close to [4].

Let $\mathbf{F}(x, \mathbf{y})=\left(F_{1}(x, \mathbf{y}), \ldots, F_{N}(x, \mathbf{y})\right)^{\prime}$ a vector ${ }^{2}$ of functions $F_{j}(x, \mathbf{y}), 1 \leq j \leq$ $N$, with complex variables $x$, and $\mathbf{y}=\left(y_{1}, \ldots, y_{N}\right)^{\prime}$ which are analytic around 0 and satisfy $F_{j}(0, \mathbf{0})=0,1 \leq j \leq N$. We will be interested in the analytic solution $\mathbf{y}=\mathbf{y}(x)=\left(y_{1}(x), \ldots, y_{N}(x)\right)^{\prime}$ of the functional equation

$$
\mathbf{y}=\mathbf{F}(x, \mathbf{y})
$$

\footnotetext{
${ }^{2}$ We always identify a $k$-dimensional vector $\mathbf{a}=\left(a_{1}, \ldots, a_{k}\right)^{\prime}$ with a $(k \times 1)$-matrix, i.e. a column. Furthermore, if $A$ is a matrix then $A^{\prime}$ denotes the transposed matrix.
} 
with $\mathbf{y}(0)=\mathbf{0}$, that is, the (unknown) functions $y_{j}=y_{j}(x), 1 \leq j \leq N$ satisfy the system of functional equations

$$
\begin{aligned}
y_{1} & =F_{1}\left(x, y_{1}, y_{2}, \ldots, y_{N}\right), \\
y_{2} & =F_{2}\left(x, y_{1}, y_{2}, \ldots, y_{N}\right), \\
& \vdots \\
y_{N} & =F_{N}\left(x, y_{1}, y_{2}, \ldots, y_{N}\right) .
\end{aligned}
$$

It is immediately clear that $y_{j}(x)$ can be represented as power series in $x$ with nonnegative coefficients $\left[x^{n}\right] y_{j}(x) \geq 0$. One only has to consider (2) as a fixed point equation and to observe that the (unique analytic) solution can be obtained by an iterating procecure with initial value $\mathbf{0}$. Of course, non-negativity of the coefficients is preserved in every iteration step.

Finally, we adjoin a dependency graph $G_{\mathbf{F}}=(V, E)$ to such a system of functional equations $\mathbf{y}=\mathbf{F}(x, \mathbf{y})$, where $V=\left\{y_{1}, y_{2}, \ldots, y_{N}\right\}$ and $\left(y_{i}, y_{j}\right)$ is contained in $E$ if and only if $F_{i}(x, \mathbf{y})$ really depends on $y_{j}$.

Lemma 4. Let $\mathbf{F}(x, \mathbf{y})=\left(F_{1}(x, \mathbf{y}), \ldots, F_{N}(x, \mathbf{y})\right)^{\prime}$ be analytic functions around $x=0$ and $\mathbf{y}=\left(y_{1}, \ldots, y_{N}\right)^{\prime}=\mathbf{0}$ such that all Taylor coefficients are non-negative, that $\mathbf{F}(0, \mathbf{y}) \equiv \mathbf{0}$, that $\mathbf{F}(x, \mathbf{0}) \not \equiv \mathbf{0}$, and that there exists $j$ with $\mathbf{F}_{y_{j} y_{j}}(x, \mathbf{y}) \not \equiv \mathbf{0}$. Furthermore assume that the region of convergence of $\mathbf{F}$ is large enough such that there exists a non-negative solution $x=x_{0}, \mathbf{y}=\mathbf{y}_{0}$ of the system of equations

$$
\begin{aligned}
& \mathbf{y}=\mathbf{F}(x, \mathbf{y}), \\
& 0=\operatorname{det}\left(\mathbf{I}-\mathbf{F}_{\mathbf{y}}(x, \mathbf{y})\right),
\end{aligned}
$$

inside it.

If the dependency graph $G_{\mathbf{F}}=(V, E)$ of the system

$$
\mathbf{y}=\mathbf{F}(x, \mathbf{y})
$$

in the unknown functions

$$
\mathbf{y}=\mathbf{y}(x)=\left(y_{1}(x), \ldots, y_{N}(x)\right)^{\prime}
$$

is strongly connected then $x_{0}$ is the common radius of convergence of $y_{1}(x), \ldots, y_{N}(x)$ and we have a representation of the form

$$
y_{j}(x)=g_{j}(x)-h_{j}(x) \sqrt{1-\frac{x}{x_{0}}}
$$

locally around $x=x_{0}$, where $g_{j}(x)$ and $h_{j}(x)$ are analytic around $x=x_{0}$ and satisfy

$$
\left(g_{1}\left(x_{0}\right), \ldots, g_{N}\left(x_{0}\right)\right)^{\prime}=\mathbf{y}_{0} \quad \text { and } \quad\left(h_{1}\left(x_{0}\right), \ldots, h_{N}\left(x_{0}\right)\right)^{\prime}=\mathbf{b}
$$

with the unique solution $\mathbf{b}=\left(b_{1}, \ldots, b_{N}\right)^{\prime}>\mathbf{0}$ of

$$
\begin{aligned}
\left(\mathbf{I}-\mathbf{F}_{\mathbf{y}}\left(x_{0}, \mathbf{y}_{0}\right)\right) \mathbf{b} & =\mathbf{0}, \\
\mathbf{b}^{\prime} \mathbf{F}_{\mathbf{y y}}\left(x_{0}, \mathbf{y}_{0}\right) \mathbf{b} & =-2 \mathbf{F}_{x}\left(x_{0}, \mathbf{y}_{0}\right) .
\end{aligned}
$$

If we further assume that $\left[x^{n}\right] y_{i}(x)>0$ for $n \geq n_{0}$ and $1 \leq j \leq N$ then $x=x_{0}$ is the only singularity of $y_{j}(x)$ on the circle $|x|=x_{0}$ and we obtain an asymptotic expansion for $\left[x^{n}\right] y_{j}(x)$ of the form

$$
\left[x^{n}\right] y_{j}(x)=\frac{b_{j}}{2 \sqrt{\pi}} x_{0}^{-n} n^{-3 / 2}\left(1+\mathcal{O}\left(n^{-1}\right)\right) .
$$


Proof. First, we consider the case of $N=1$ equation:

$$
y=F(x, y) .
$$

As mentioned above it is clear that the coefficients of the (unique) solution $y(x)$ (that is obtained by iteration) are non-negative.

It is also possible to use the implicit function theorem. Since

$$
F_{y}(0,0)=0 \neq 1 \text {. }
$$

there exists a solution $y=y(x)$ of $(7)$ which is analytic around 0 .

However, it is useful to know that all Taylor coefficients of $y(x)$ are non-negative. Namely, it follows that if $y(x)$ is regular at $x^{\prime}$ (that is real and positive) then $y(x)$ is regular for all $x$ with $|x| \leq x^{\prime}$.

Let $x_{0}$ denote the radius of convergence of $y(x)$. Then $x_{0}$ is a singularity of $y(x)$. The mapping

$$
x \mapsto F_{y}(x, y(x))
$$

is strictly increasing for real and non-negative $x$ as long as $y(x)$ is regular. Note that $F_{y}(0, y(0))=0$. As long as $F_{y}(x, y(x))<1$ it follows from the implicit function theorem that $y(x)$ is regular even in a neighbourhood of $x$. Hence there exists a finite limit point $x_{0}$ such that $\lim _{x \rightarrow x_{0}-} y(x)=y_{0}$ is finite and satisfies $F_{y}\left(x_{0}, y_{0}\right)=1$. If $y(x)$ were regular at $x=x_{0}$ then

$$
y^{\prime}\left(x_{0}\right)=F_{x}\left(x_{0}, y(x)\right)+F_{y}\left(x_{0}, y\left(x_{0}\right)\right) y^{\prime}\left(x_{0}\right)
$$

would imply $F_{x}\left(x_{0}, y\left(x_{0}\right)\right)=0$ which is surely not true. Thus, $y(x)$ is singular at $x=x_{0}$ (that is, $x_{0}$ is the radius of convergence) and $y\left(x_{0}\right)$ is finite.

Now, let us consider the equation $y-F(x, y)=0$ around $x=x_{0}$ and $y=y_{0}$. We have $1-F_{y}\left(x_{0}, y_{0}\right)=0$ but $-F_{y y}\left(x_{0}, y_{0}\right) \neq 0$. Hence by the Weierstrass preparation theorem (see [7]) there exist functions $H(x, y), p(x), q(x)$ which are analytic around $x=x_{0}$ and $y=y_{0}$ and satisfy $H\left(x_{0}, y_{0}\right) \neq 1, p\left(x_{0}\right)=q\left(x_{0}\right)=0$ and

$$
y-F(x, y)=H(x, y)\left(\left(y-y_{0}\right)^{2}+p(x)\left(y-y_{0}\right)+q(x)\right)
$$

locally around $x=x_{0}$ and $y=y_{0}$. Since $F_{x}\left(x_{0}, y_{0}\right) \neq 0$ we also have $q_{x}\left(x_{0}\right) \neq 0$. This means that any analytic function $y=y(x)$ which satisfies $y(x)=F(x, y(x))$ in a subset of a neighbourhood of $x=x_{0}$ with $x_{0}$ on its boundary and is given by

$$
y(x)=y_{0}-\frac{p(x)}{2} \pm \sqrt{\frac{p(x)^{2}}{4}-q(x)} .
$$

Since $p\left(x_{0}\right)=0$ and $q_{x}\left(x_{0}\right) \neq 0$ we have

$$
\frac{\partial}{\partial x}\left(\frac{p(x)^{2}}{4}-q(x)\right)_{x=x_{0}} \neq 0,
$$

too. Thus there exist an analytic function $K(x)$ such that $K\left(x_{0}\right) \neq 0$ and

$$
\frac{p(x)^{2}}{4}-q(x)=K(x)\left(x-x_{0}\right)
$$

locally around $x=x_{0}$. This finally leads to a local representation of $y=y(x)$ of the kind

$$
y(x)=g(x)-h(x) \sqrt{1-\frac{x}{x_{0}}},
$$

in which $g(x)$ and $h(x)$ are analytic around $x=x_{0}$ and satisfy $g\left(x_{0}\right)=y_{0}$ and $h\left(x_{0}\right)<0$. 
In order to calculate $h\left(x_{0}\right)$ we use Taylor's theorem

$$
\begin{aligned}
0 & =F(x, y(x)) \\
& =F_{x}\left(x_{0}, y_{0}\right)\left(x-x_{0}\right)+\frac{1}{2} F_{y y}\left(x_{0}, y_{0}\right)\left(y(x)-y_{0}\right)^{2}+\cdots \\
& =F_{x}\left(x_{0}, y_{0}\right)\left(x-x_{0}\right)+\frac{1}{2} F_{y y}\left(x_{0}, y_{0}\right) h\left(x_{0}\right)^{2}\left(1-x / x_{0}\right)+\mathcal{O}\left(\left|x-x_{0}\right|^{3 / 2}\right) .
\end{aligned}
$$

By comparing the coefficients of $\left(x-x_{0}\right)$ we immediately obtain

$$
h\left(x_{0}\right)=\sqrt{\frac{2 x_{0} F_{x}\left(x_{0}, y_{0}\right)}{F_{y y}\left(x_{0}, y_{0}\right)}} .
$$

We now want to apply the transfer lemma (Lemma 1). For this purpose we have to show that $y(x)$ can be analytically continued to a region of the form $\Delta$. The representation (8) provides such an analytic continuation for $x$ in a neighborhood of $x_{0}$. Now suppose that $\left|x_{1}\right|=x_{0}$ and $\left|\arg \left(x_{1}\right)\right| \geq \delta$. Then the assumption $y_{n}>0$ for $n \geq n_{0}$ implies that $\left|y\left(x_{1}\right)\right|<y\left(\left|x_{1}\right|\right)=y\left(x_{0}\right)$ and consequently

$$
\left|F_{y}\left(x_{1}, y\left(x_{1}\right)\right)\right| \leq F_{y}\left(\left|x_{1}\right|,\left|y\left(x_{1}\right)\right|\right)<F_{y}\left(\left|x_{1}\right|, y\left(\left|x_{1}\right|\right)\right)=F_{y}\left(x_{0}, y_{0}\right)=1 .
$$

Thus, $F_{y}\left(x_{1}, y\left(x_{1}\right)\right) \neq 1$ and the implicit function theorem shows that there exists an analytic solution $y=y(x)$ in a neighborhood of $x_{1}$. For $|x|<x_{0}$ this solution equals the power series $y(x)$ and for $|x| \geq x_{0}$ it provides an analytic continuation to a region of the form $\Delta$ (by compactness it is sufficient to consider finitely many $x_{1}$ with $\left|x_{1}\right|=x_{0}$ and $\left|\arg \left(x_{1}\right)\right| \geq \delta$ ). So finally we can apply Lemma 1 (resp. (1) with $\alpha=-1 / 2$ and $\beta=-3 / 2$; the analytic part of $g(x)$ provides exponentially smaller contributions.) This completes the proof of (6).

Finally we mention that the general case $N>1$ can be reduced to a single equation by an elimination precedure (compare to [4]). We will indicate this procedure for $N=2$ equations. $y_{1}=F_{1}\left(x, y_{1}, y_{2}\right), y_{2}=F_{2}\left(x, y_{1}, y_{2}\right)$. First, we tackle the second equation and consider $x$ and $y_{1}$ as variables. This gives $y_{2}=y_{2}\left(x, y_{1}\right)$ as a power series in non-negative coefficients in $x$ and $y_{1}$. Next we use this "solution" an insert it into the first equation. Thus, we obtain a single equation

$$
y_{1}=F_{1}\left(x, y_{1}, y_{2}\left(x, y_{1}\right)\right)
$$

that can be treated as above (all assumptions are satisfied). The singularity $x_{0}$ of $y_{1}(x)$ is determined by the equation

$$
1=F_{1, y_{1}}+F_{1, y_{2}} y_{2, y_{1}}=F_{1, y_{1}}+F_{1, y_{2}} \frac{F_{2, y_{1}}}{1-F_{2, y_{2}}}
$$

or by

$$
\left(1-F_{1, y_{1}}\right)\left(1-F_{2, y_{2}}\right)-F_{1, y_{2}} F_{2, y_{1}}=0 .
$$

Of course, this procedure only applies is $y_{2}\left(x, y_{1}\right)$ is not singular, respectively $F_{2, y_{2}}<1$. Since we assume that the dependency graph is strongly connected we surely have $F_{1, y_{2}} \neq 0$ and $F_{2, y_{1}} \neq 0$. Thus, must have $F_{2, y_{2}}<1$ at the singular point of $y_{1}(x)$, in other words $y_{2}\left(x, y_{1}\right)$ is regular there and does not "effect" the singularity of $y_{1}(x)$. However, we can now use $y_{1}(x)$ to "recover" the "real solution" $y_{2}(x)=y_{2}\left(x, y_{1}(x)\right)$ that has the same kind of singular behaviour as $y_{1}(x)$. 
2.3. Small Powers of Functions. The next lemma shown how (small) powers of functions with a squareroot singularity can be handled, see also [3].

Lemma 5. Let $y(x)=\sum_{n \geq 0} y_{n} x^{n}$ be a power series with non-negative coefficents such that there is only one singularity on the circle of convergence $|x|=x_{0}>0$ and that $y(x)$ can be locally represented as

$$
y(x)=g(x)-h(x) \sqrt{1-\frac{x}{x_{0}}},
$$

where $g(x)$ and $h(x)$ are analytic functions around $x_{0}$ with $g\left(x_{0}\right)>0$ and $h\left(x_{0}\right)>0$, and that $y(x)$ can be continued analytically to $|x|<x_{0}+\delta, x \notin\left[x_{0}, x_{0}+\delta\right.$ ) (for some $\delta>0)$. Furthermore, let $\rho(x)$ be another power series with non-negative coefficients with radius of convergence greater than $x_{0}$.

Then we have

$$
\left[x^{n}\right] \rho(x) y(x)^{k}=\frac{k \rho\left(x_{0}\right) g\left(x_{0}\right)^{k-1} h\left(x_{0}\right)}{2 n^{\frac{3}{2}} \sqrt{\pi} x_{0}^{n}}\left(\exp \left(-\frac{k^{2}}{4 n}\left(\frac{h\left(x_{0}\right)}{g\left(x_{0}\right)}\right)^{2}\right)+\mathcal{O}\left(\frac{k}{n}\right)\right)
$$

uniformly for $k \leq C \sqrt{n}$, where $C>0$ is an arbitrary constant.

Proof. For simplicity we will assume that the radius of convergence $x_{0}=1$. We will use Cauchy's formula

$$
\left[x^{n}\right] \rho(x) y(x)^{k}=\frac{1}{2 \pi i} \int_{\gamma} \rho(x) y(x)^{k} x^{-n-1} d x,
$$

where the path of integration $\gamma=\gamma_{1} \cup \gamma_{2} \cup \gamma_{3} \cup \gamma_{4}$ is the same as in the proof of Lemma 2.

Let $k \leq C \sqrt{n}$. Then with $x=1+\frac{t}{n}$ we have for $x \in \gamma_{1} \cup \gamma_{2} \cup \gamma_{3}$

$$
\begin{aligned}
\rho(x) y(x)^{k} x^{-(n+1)=} & \rho(x) g(x)^{k}\left(1-\frac{h(x)}{g(x)} \sqrt{1-x}\right)^{k} x^{-(n+1)} \\
= & \rho(1) g(1)^{k} \exp \left(-\frac{k}{\sqrt{n}} \frac{h(1)}{g(1)}(-t)^{\frac{1}{2}}-t\right) . \\
& \cdot\left(1+\mathcal{O}\left(\frac{|t|^{2}}{n}\right)+\mathcal{O}\left(\frac{k|t|}{n}\right)+\mathcal{O}\left(k \frac{|t|^{\frac{3}{2}}}{n^{\frac{3}{2}}}\right)\right) .
\end{aligned}
$$

Let $\lambda=\frac{k}{\sqrt{n}} \frac{h(1)}{g(1)}$. Then (with help of the substitution $u^{2}=t$ ) it is an easy exercise to obtain

$$
\frac{1}{2 \pi i} \int_{\gamma^{\prime}} e^{-\lambda \sqrt{-t}-t} d t=\frac{\lambda}{2 \sqrt{\pi}} e^{-\frac{\lambda^{2}}{4}}+\mathcal{O}\left(e^{-\log ^{2} n}\right),
$$

where $\gamma^{\prime}=\{t|| t \mid=1, \Re t \leq 0\} \cup\left\{t \mid 0<\Re t \leq \log ^{2} n\right.$, ऽt $\left.= \pm 1\right\}$ is the same path of integration as used in Lemma 2.

Furthermore, for every fixed $L$ we have

$$
\int_{\gamma^{\prime}}\left|e^{-\lambda \sqrt{-t}-t} t^{L}\right| d t=\mathcal{O}(1)
$$

All these estimates are also uniform if $\lambda$ varies in an bounded interval. Finally,

$$
\int_{\gamma_{4}} \rho(x) y(x)^{k} x^{-n-1} d x=\mathcal{O}\left(\rho(1) g(1)^{k} e^{-\left(1-\frac{k}{n} \frac{h(1)}{g(1)}\right) \log ^{2} n}\right) .
$$

This completes the proof of Lemma 5 . 
The next lemma is very similar to Lemma 5 . The only difference is that the leading factor $\rho(x)$ also has a special squareroot singularity. In particular, this lemma will be used for null recurrent QBD's (second parts of Theorem 1 and 2).

Lemma 6. Let $y(x)=\sum_{n \geq 0} y_{n} x^{n}$ be as in Lemma 5 and $\rho(x)$ another power series that has the same radius of convergence $x_{0}$ that it can be continued analytically to the same region as $y(x)$, and that it has a local (singular) represenation as

$$
\rho(x)=\frac{\bar{g}(x)}{\sqrt{1-\frac{x}{x_{0}}}}+\bar{h}(x)
$$

where $\bar{g}(x)$ and $\bar{h}(x)$ are analytic functions around $x_{0}$ with $\bar{g}\left(x_{0}\right)>0$.

Then we have

$$
\left[x^{n}\right] \rho(x) y(x)^{k}=\frac{\bar{g}\left(x_{0}\right) g\left(x_{0}\right)^{k}}{\sqrt{n \pi} x_{0}^{n}}\left(\exp \left(-\frac{k^{2}}{4 n}\left(\frac{h\left(x_{0}\right)}{g\left(x_{0}\right)}\right)^{2}\right)+\mathcal{O}\left(\frac{k}{n}\right)\right)
$$

uniformly for $k \leq C \sqrt{n}$, where $C>0$ is an arbitrary constant.

Proof. The proof is almost the same as that of Lemma 5. The only difference is that one has to use the formula

$$
\frac{1}{2 \pi i} \int_{\gamma^{\prime}} \frac{e^{-\lambda \sqrt{-t}-t}}{\sqrt{-t}} d t=\frac{1}{\sqrt{\pi}} e^{-\lambda^{2} / 4}+\mathcal{O}\left(e^{-(\log n)^{2}}\right) .
$$

We leave the detail to the reader.

2.4. Large Powers of Functions. Finally we consider large powers of power series, that is, $n$ and $k$ are proportional (see $[2,3]$ ).

We start with a general formula.

Lemma 7. Let $y(x)=\sum_{n \geq 0} y_{n} x^{n}$ be a power series with non-negative coefficents, moreoever, assume that there exists $n_{0}$ with $y_{n}>0$ for $n \geq n_{0}$. Furthermore, let $\rho(x)$ be another power series with non-negative coefficients and suppose that, both, $y(x)$ and $\rho(x)$ have positive radius of convergence $R_{1}, R_{2}$. Set

$$
\mu(r)=\frac{r y^{\prime}(r)}{y(r)}
$$

and

$$
\sigma^{2}(r):=r \mu^{\prime}(r)=\frac{r y^{\prime}(r)}{y(r)}+\frac{r^{2} y^{\prime \prime}(r)}{y(r)}-\frac{r^{2} y^{\prime}(r)^{2}}{y(r)^{2}}
$$

and let $h(y)$ denote the inverse function of $\mu(r)$.

Fix $a, b$ with $0<a<b<\min \left\{R_{1}, R_{2}\right\}$, then we have

$$
\left[x^{n}\right] \rho(x) y(x)^{k}=\frac{1}{\sqrt{2 \pi k}} \frac{\rho\left(h\left(\frac{n}{k}\right)\right)}{\sigma\left(h\left(\frac{n}{k}\right)\right)} \frac{y\left(h\left(\frac{n}{k}\right)\right)^{k}}{h\left(\frac{n}{k}\right)^{n}} \cdot\left(1+\mathcal{O}\left(\frac{1}{k}\right)\right)
$$

uniformly for $n, k$ with $\mu(a) \leq n / k \leq \mu(b)$.

Proof. We (again) use Cauchy's formula

$$
\left[x^{n}\right] \rho(x) y(x)^{k}=\frac{1}{2 \pi i} \int_{|x|=r} \rho(x) y(x)^{k} x^{-n-1} d x
$$


where $r$ is defined by

$$
\frac{r y^{\prime}(r)}{y(r)}=\frac{n}{k}
$$

that is, $r=h\left(\frac{n}{k}\right)$. Note that $r$ is exactly the saddle point of the function

$$
y(x)^{k} x^{-n}=e^{k \log y(x)-n \log x} .
$$

Now a standard saddle point method (see [3] or [12]) yields

$$
\left[x^{n}\right] \rho(x) y(x)^{k}=\frac{\rho(r) y(r)^{k} r^{-n}}{\sqrt{2 \pi k \sigma^{2}(r)}} \cdot\left(1+\mathcal{O}\left(\frac{1}{k}\right)\right) .
$$

In detail, if we use the substituion $x=r e^{i t}$ we get (for small $|t| \leq k^{-\frac{1}{2}+\eta}$ with $\left.0<\eta<\frac{1}{6}\right)$

$$
\rho(x) y(x)^{k} x^{-n}=\rho(r) y(r)^{k} r^{-n} e^{-k t^{2} \sigma^{2}(r)+\mathcal{O}\left(|t|+k|t|^{3}\right)}
$$

and consequently

$$
\frac{1}{2 \pi i} \int_{|t| \leq k^{-\frac{1}{2}+\eta}} \rho(x) y(x)^{k} x^{-n-1} d x=\frac{\rho(r) y(r)^{k} r^{-n}}{\sqrt{2 \pi k \sigma^{2}(r)}} \cdot\left(1+\mathcal{O}\left(\frac{1}{k}\right)\right) .
$$

Finally, by the use of the property that $y_{n}>0$ (for $n \geq n_{0}$ ) and the local expansion of $y(x)$ around $x=r$ it follows that there exists a constant $c>0$ with

$$
\left|y\left(r e^{i t}\right)\right| \leq y(r) e^{-c t^{2}}
$$

uniformly for $a \leq r \leq b$ and $|t| \leq \pi$. Hence the integral

$$
\frac{1}{2 \pi i} \int_{k^{-\frac{1}{2}+\eta} \leq|t| \leq \pi} \rho(x) y(x)^{k} x^{-n-1} d x=\mathcal{O}\left(\rho(r) y(r)^{k} r^{-n} e^{-c k^{2 \eta}}\right)
$$

is negligible.

We can also obtain a slightly modified version of Lemma 7 (compare with [3]) if $n$ and $k$ are almost proprotional. This can be either derived from Lemma 7 or proved in a completely similar way. We leave the details to the reader.

Lemma 8. Let $y(x)$ and $\rho(x)$ be as in Lemma 7. Then for every $0<r<$ $\min \left\{R_{1}, R_{2}\right\}$ we have

$$
\left[x^{n}\right] \rho(x) y(x)^{k}=\frac{1}{\sqrt{2 \pi k}} \frac{\rho(r)}{\sigma(r)} \frac{y(r)^{k}}{r^{n}} \cdot\left(\exp \left(-\frac{(k-n / \mu(r))^{2}}{2 k \sigma^{2}(r)}\right)+\mathcal{O}\left(\frac{1}{\sqrt{n}}\right)\right)
$$

uniformly for $n, k$ with $|k-n / \mu(r)| \leq C \sqrt{k}$, where $C>0$ is an arbitrary constant.

Remark . It should be remarked that it is also possible to derive complete asymptotic series expansion, see [3]. 


\section{Quasi Birth and Death Processes}

3.1. Random Walks on "Perodic Graphs". We now consider a discrete quasi birth and death process (QBD), that is, a discrete Markov process $X_{n}$ on the nonnegative integers with transition matrix of the form

$$
\mathbf{P}=\left(\begin{array}{cccccc}
\mathbf{B} & \mathbf{A}_{0} & \mathbf{0} & \mathbf{0} & \ldots & \\
\mathbf{A}_{2} & \mathbf{A}_{1} & \mathbf{A}_{0} & \mathbf{0} & \ldots & \\
\mathbf{0} & \mathbf{A}_{2} & \mathbf{A}_{1} & \mathbf{A}_{0} & \mathbf{0} & \ldots \\
\mathbf{0} & \mathbf{0} & \mathbf{A}_{2} & \mathbf{A}_{1} & \mathbf{A}_{0} & \ldots \\
\vdots & & \ddots & \ddots & \ddots & \ddots
\end{array}\right)
$$

where $\mathbf{A}_{0}, \mathbf{A}_{1}, \mathbf{A}_{2}$, and $\mathbf{B}$ are square matrices of order $m$, compare with $[9,10,11]$.

This kind of process can be also interpreted as a random walks on infinite graphs $G$ of the following type (see [5]). Let $K$ be the complete di-graphs of size $m$ (with loops) and $K_{0}, K_{1}, K_{2}, \ldots$ copies of $K$. The set of nodes, $V(G)$, of $G$ is now given by $V\left(K_{0}\right) \cup V\left(K_{1}\right) \cup \cdots$. The directed edges of $G, E(G)$, consist first of the edges $E\left(K_{0}\right) \cup E\left(K_{1}\right) \cup \cdots$ and second of all edges between $K_{0}$ and $K_{1}$, between $K_{1}$ and $K_{2}$ etc. We denote the matrix of transition probabilities inside $K_{0}$ as $\mathbf{B}$, inside $K_{j}$ $(j=1,2, \ldots)$ as $\mathbf{A}_{1}$, between $K_{j}$ and $K_{j+1}$ as $\mathbf{A}_{0}$, and between $K_{j}$ and $K_{j-1}$ as $\mathbf{A}_{2}$ (see figure 1).

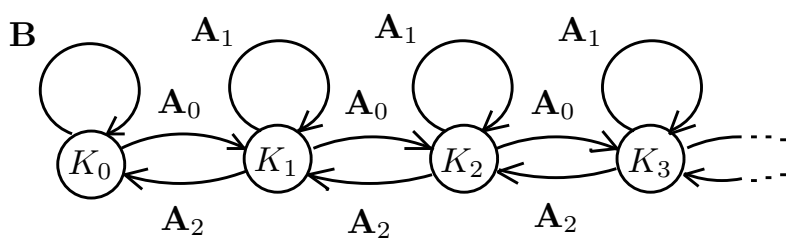

FiguRE 1. One-sided "periodic" graph

In other words, if we set $\mathbf{P}=\left(p_{w, v}\right)_{w, v \in V(G)}$, then (for all $k \geq 0$ )

$$
p_{w, v}=\operatorname{Pr}\left\{X_{k+1}=v \mid X_{k}=w\right\} .
$$

It is clear that the powers $\mathbf{P}^{n}=\left(p_{w, v}^{(n)}\right)_{w, v \in V(G)}$ of the infinite matrix $\mathbf{P}$ contain the probabilities $p_{w, v}^{(n)}=\operatorname{Pr}\left\{X_{n}=v \mid X_{0}=w\right\}$.

We will now present a combinatorial interpretation of this relation. Let $\tilde{p}=$ $\left(e_{1}(\tilde{p}), e_{2}(\tilde{p}), \ldots, e_{n}(\tilde{p})\right)$ denotes a path on length $n$ on $G$ (with edges $e_{j}(\tilde{p})=$ $\left.\left(x_{j-1}(\tilde{p}), x_{j}(\tilde{p})\right) \in E(G)\right)$. Then we can define a weight (or probability) $W(\tilde{p})$ by

$$
W(\tilde{p})=\prod_{j=1}^{n} p_{\left(x_{j-1}(\tilde{p}), x_{j}(\tilde{p})\right)}
$$

and, of course, we get

$$
p_{w, v}^{(n)}=\sum W(\tilde{p})
$$

where the sum is taken over all paths $\tilde{p}$ of length $n$ with $x_{0}(\tilde{p})=w$ and $x_{n}(\tilde{p})=v$. This means that the calculation of $p_{w, v}^{(n)}$ can be viewed as a combinatorial enumeration problem of weighted paths of length $n$. In this context it is natural to introduce 
the power series (of generating functions)

$$
M_{w, v}(x)=\sum_{n \geq 0} p_{w, v}^{(n)} x^{n} .
$$

The (infinite) matrix $\mathbf{M}(x)=\left(M_{w, v}(x)\right)_{w, v \in V(G)}$ can be then viewed as

$$
\mathbf{M}(x)=(\mathbf{I}-x \mathbf{P})^{-1} .
$$

In what follows we will use the combinatorial interpretation to derive explicit formulae for (the first $m$ rows of) $\mathbf{M}(x)$.

For this purpose we also define proper submatrices of order $m$

$$
\mathbf{M}_{k, \ell}(x)=\left(M_{w, v}(x)\right)_{w \in K_{k}, v \in K_{\ell}}
$$

that correspond to the transition from $K_{k}$ to $K_{\ell}$.

Since we are only interested in processes $X_{n}$ starting in $K_{0}$ we will only state a result for $\mathbf{M}_{0, \ell}(x)$. However, it is an easy exercice to derive formulae for all $\mathbf{M}_{k, \ell}(x)$, compare with the remark after the proof of Lemma 9.

Lemma 9. Let $\mathbf{N}(x)$ denote the (analytic) solution with $\mathbf{N}(0)=\mathbf{I}$ of the matrix equation

$$
\mathbf{N}(x)=\mathbf{I}+x \mathbf{A}_{1} \mathbf{N}(x)+x^{2} \mathbf{A}_{0} \mathbf{N}(x) \mathbf{A}_{2} \mathbf{N}(x) .
$$

Then

$$
\mathbf{M}_{0, \ell}(x)=x^{\ell}\left(\mathbf{I}-x \mathbf{B}-x^{2} \mathbf{A}_{0} \mathbf{M}(x) \mathbf{A}_{2}\right)^{-1}\left(\mathbf{A}_{0} \mathbf{N}(x)\right)^{\ell} .
$$

Proof. In order to make the situation simpler we will first consider the case $m=1$, that is, the corresponding graph is just the line of non-negative integers (and the transition matrix $\mathbf{P}$ is a tri-diagonal matrix). We will heavily make use of the combinatorial description of with help of weighted paths $\tilde{p}$.

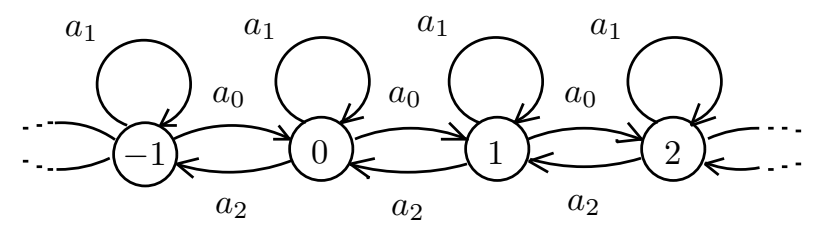

FiguRE 2. Random walk on the integers

We start with a property of one-sided paths on the integers (see Figure 2). Let $Y_{n}$ denote the random walk on the integers (see Figure 2) with $Y_{0}=0$. For this random walk we consider the generating function of one-sided return probabilities

$$
N(x)=\sum_{n \geq 0} \operatorname{Pr}\left\{Y_{1} \geq 0, Y_{2} \geq 0, \ldots, Y_{n-1} \geq 0, Y_{n}=0 \mid Y_{0}=0\right\} \cdot x^{n} .
$$

Of course, the probabilities can be interpreted as sums of weights. First we show that $N(x)$ satisfies the functional equation

$$
N(x)=1+a_{1} x N(x)+a_{0} a_{2} x^{2} N(x)^{2}
$$

This equation is immedialtely clear by writing it in slightly different way:

$$
N(x)=1+a_{1} x \cdot N(x)+a_{0} x \cdot N(x) \cdot a_{2} x \cdot N(x) ;
$$


and by looking at the following recursive description. First, 1 is related to the case $n=0$. Next, if the first step of the path is a loop (with probability $a_{1}$ ) then the remaining part is just a non-negative path from 0 to 0 , the corresponding contribution is $a_{1} x \cdot M(x)$. If the first step goes to the right (with probability $a_{0}$ ) then we decompose the path into four parts: into this first step from 0 to the right, into a part from 1 to 1 that is followed by the first step back from 1 to 0 , the third part is this step back, and finally into the last part that is again a non-negative path from 0 to 0 . Hence, in terms of generating functions this case contributed $a_{0} x \cdot N(x) \cdot a_{2} x \cdot N(x)$. This completes the proof of (14).

With help of the same reasoning one gets the relation

$$
M_{0,0}(x)=1+b x M_{0,0}(x)+a_{0} x N(x) a_{2} x M_{0,0}(x)
$$

that proves the proposed representation for $M_{0,0}(x)$.

Next we have $M_{0,1}(x)=M_{0,0}(x) a_{0} x N(x)$. Here we have to divide all paths from 0 to 1 into three parts. The first part is just the path from 0 to 0 that is followed by the last step from 0 to 1 . This step is the second part, and the third part is a non-negative path from 1 to 1 . In a similar way we also obtain the recurrence $M_{0, \ell+1}(x)=M_{0, \ell}(x) a_{0} x N(x)$.

The proof in the general case $(m>1)$ is exactly the same and already appears (for a special case) in [13], compare also with [8]. We first consider a two-sided infinite periodic graph (containing components $K_{\ell}, \ell \in \mathbb{Z}$ and random walks $Y_{n}$ that start in $K_{0}$ and do not go to the negative part). This leads to a matrix generating function $\mathbf{N}(x)$ that satisfies (11). And with help of $\mathbf{N}(x)$ we directly get representations for $\mathbf{M}_{0, \ell}(x)$ :

$$
\mathbf{M}_{0,0}(x)=\mathbf{I}+\mathbf{B} x \mathbf{M}_{0,0}(x)+\mathbf{A}_{0} x \mathbf{N}(x) \mathbf{A}_{2} x \mathbf{M}_{0,0}(x)
$$

and

$$
\mathbf{M}_{0, \ell+1}(x)=\mathbf{M}_{0, \ell}(x) \mathbf{A}_{0} x \mathbf{N}(x) .
$$

Of course, this proves (12).

Remark - As already indicated it is also possible to get explicit formulae for all matrices $\mathbf{M}_{k, \ell}(x)$. We just state corresponding recurrences from which these representation can be derived:

$\mathbf{M}_{\ell+1, \ell+1}(x)=\mathbf{I}+\mathbf{A}_{1} x \mathbf{M}_{\ell+1, \ell+1}(x)+\mathbf{A}_{0} x \mathbf{N}(x) \mathbf{A}_{2} x \mathbf{M}_{\ell+1, \ell+1}(x)+\mathbf{A}_{2} x \mathbf{M}_{\ell, \ell}(x) \mathbf{A}_{0} x \mathbf{N}(x)$

or

$$
\mathbf{M}_{\ell+1, \ell+1}(x)=\left(\mathbf{I}-x \mathbf{A}_{1}-x^{2} \mathbf{A}_{0} \mathbf{M}(x) \mathbf{A}_{2}\right)^{-1}\left(\mathbf{I}+\mathbf{A}_{2} x \mathbf{M}_{\ell, \ell}(x) \mathbf{A}_{0} x \mathbf{N}(x)\right),
$$

further

and

$$
\mathbf{M}_{k, k+\ell+1}(x)=\mathbf{M}_{k, k+\ell}(x) \mathbf{A}_{0} x \mathbf{N}(x),
$$

$$
\mathbf{M}_{k+\ell+1, \ell}(x)=\mathbf{N}(x) \mathbf{A}_{2} x \mathbf{M}_{k+\ell, \ell}(x) .
$$

Remark . Lemma 9 is contained in [5] in a slightly more general form.

Note further that the generating function $N(x)$ is closely related to the generating functions $U(x), G(x)$, and $R(x)$ presented in [9, p. 96]. We have $N(x)=1 /(1-$ $U(x)), G(x)=M(x) \cdot d x$, and $R(x)=c x \cdot M(x)$.

Moreoever, for $x=1$ the matrix $\mathbf{N}=\mathbf{N}(1)$ is also related to the matrices $\mathbf{U}, \mathbf{G}$, and $\mathbf{R}$ of [9, p. 137], in particular, $\mathbf{N}=(\mathbf{I}-\mathbf{U})^{-1}, \mathbf{G}=\mathbf{N D}$, and $\mathbf{R}=\mathbf{C N}$. 
3.2. Continuous Quasi Birth and Death Processes. There exists a continuous analogon the discrete QBD. Here we have a continuous time Markov process $X(t)$ on $G$ with infinitesimal generator $\mathbf{Q}$ of the same form

$$
\mathbf{Q}=\left(\begin{array}{cccccc}
\mathbf{B} & \mathbf{A}_{0} & \mathbf{0} & \mathbf{0} & \ldots & \\
\mathbf{A}_{2} & \mathbf{A}_{1} & \mathbf{A}_{0} & \mathbf{0} & \ldots & \\
\mathbf{0} & \mathbf{A}_{2} & \mathbf{A}_{1} & \mathbf{A}_{0} & \mathbf{0} & \ldots \\
\mathbf{0} & \mathbf{0} & \mathbf{A}_{2} & \mathbf{A}_{1} & \mathbf{A}_{0} & \ldots \\
\vdots & & \ddots & \ddots & \ddots & \ddots
\end{array}\right)
$$

where $\mathbf{A}_{0}, \mathbf{A}_{1}, \mathbf{A}_{2}$, and $\mathbf{B}$ are square matrices of order $m$ such that $\mathbf{A}_{0}$ and $\mathbf{A}_{2}$ are non-negative and the matrices $\mathbf{B}$ and $\mathbf{A}_{1}$ have non-negative off-diagonal elements whereas the diagonal elements are stricly negative so that the row sums are all equal to zero, that is, $\left(\mathbf{B}+\mathbf{A}_{0}\right) \mathbf{1}=\mathbf{0}$ and $\left(\mathbf{A}_{0}+\mathbf{A}_{1}+\mathbf{A}_{2}\right) \mathbf{1}=\mathbf{0}$.

By definition it is clear that the matrix $e^{\mathbf{Q} t}=\left(q_{w, v}^{(t)}\right)_{w, v \in G}$ contains the probabilities

$$
q_{w, v}^{(t)}=\operatorname{Pr}\{X(t)=v \mid X(0)=w\} .
$$

Similarly to the discrete case we now consider the Laplace transforms

$$
\hat{M}_{w, v}(s)=\int_{0}^{\infty} \operatorname{Pr}\{X(t)=v \mid X(0)=w\} e^{-s t} d t
$$

and the infite matrix $\hat{\mathbf{M}}(s)=\left(\hat{M}_{w, v}(s)\right)_{w, v \in V(G)}$. By the above property we can rewrite $\hat{\mathbf{M}}(s)$ as

$$
\hat{\mathbf{M}}(s)=(s \mathbf{I}-\mathbf{Q})^{-1} .
$$

This means that $\hat{\mathbf{M}}(s)$ has almost the same representation as $\mathbf{M}(x)$ in the discrete case. Consequently we also get (almost) the same explicit representation for

$$
\hat{\mathbf{M}}_{k, \ell}(s)=\left(\hat{M}_{w, v}(s)\right)_{w \in K_{k}, v \in K_{\ell}} .
$$

Lemma 10. Let $\mathbf{N}(x)$ denote the (analytic) solution with $\mathbf{N}(0)=\mathbf{I}$ of the matrix equation

$$
\mathbf{N}(x)=\mathbf{I}+x \mathbf{A}_{1} \mathbf{N}(x)+x^{2} \mathbf{A}_{0} \mathbf{N}(x) \mathbf{A}_{2} \mathbf{N}(x) .
$$

Then

$$
\hat{\mathbf{M}}_{0, \ell}(s)=\left(s \mathbf{I}-\mathbf{B}-\mathbf{A}_{0} \frac{1}{s} \mathbf{N}\left(\frac{1}{s}\right) \mathbf{A}_{2}\right)^{-1}\left(\mathbf{A}_{0} \frac{1}{s} \mathbf{N}\left(\frac{1}{s}\right)\right)^{\ell} .
$$

Remark . Note that $\hat{\mathbf{N}}(s):=\frac{1}{s} \mathbf{N}\left(\frac{1}{s}\right)$ can be also characterized by

$$
s \hat{\mathbf{N}}(s)=\mathbf{I}+\mathbf{A}_{1} \hat{\mathbf{N}}(s)+\mathbf{A}_{0} \hat{\mathbf{N}}(s) \mathbf{A}_{2} \hat{\mathbf{N}}(s)
$$

with $\lim _{s \rightarrow \infty} s \hat{\mathbf{N}}(s)=\mathbf{I}$ and $\mathbf{M}_{0, \ell}(s)$ is then given by

$$
\hat{\mathbf{M}}_{0, \ell}(s)=\left(s \mathbf{I}-\mathbf{B}-\mathbf{A}_{0} \hat{\mathbf{N}}(s) \mathbf{A}_{2}\right)^{-1}\left(\mathbf{A}_{0} \hat{\mathbf{N}}(s)\right)^{\ell} .
$$

Proof. We can proceed in a formal way. First we define weights $W(\tilde{p})$ for paths on $G$ with help of the infinite generator matrix $\mathbf{Q}$ and obtain representations for 
generating functions that "count" weighted paths or, equivalently, explicit representations of the first $m$ rows of the matrix $\mathbf{M}(x)=(\mathbf{I}-x \mathbf{Q})^{-1}$. However, the Laplace transform of interest is given by

$$
\hat{\mathbf{M}}(s)=(s \mathbf{I}-\mathbf{Q})^{-1}=\frac{1}{s}\left(\mathbf{I}-\frac{1}{s} \mathbf{Q}\right)^{-1} .
$$

Consequently, $\hat{\mathbf{M}}_{k, \ell}(s)=\frac{1}{s} \mathbf{M}_{k, \ell}\left(\frac{1}{s}\right)$. Thus, Lemma 17 follows (formally) from Lemma 12.

\section{The Asymptotic Behaviour of Homogeneous QBD's}

In this section we apply the asymptotic enumeration methods of section 2 to homoegeneous QBD's. The discrete case has been already treated by the author [5]. Furthermore, some parts of the results are definitely not new, e.g. the positive recurrent case is very well studied, see [9]. Nevertheless, it seems that the kind of approch is novel.

4.1. One-Dimensional Discrete QBD's. We start with the most easy example, namely with one-dimensional QBD's that can be interpreted as random walks on the non-negative integers.

Theorem 1. Suppose that $a_{0}, a_{1}, a_{2}$ and $b$ are positive numbers with $a_{0}+a_{1}+a_{2}=$ $b+a_{0}=1$; and let $X_{n}$ be the discrete $Q B D$ on the non-negative integers with transition matrix

$$
\mathbf{P}=\left(\begin{array}{cccccc}
b & a_{0} & 0 & 0 & \ldots & \\
a_{2} & a_{1} & a_{0} & 0 & \ldots & \\
0 & a_{2} & a_{1} & a_{0} & 0 & \ldots \\
0 & 0 & a_{2} & a_{1} & a_{0} & \ldots \\
\vdots & & \ddots & \ddots & \ddots & \ddots
\end{array}\right)
$$

(1) If $a_{0}<a_{2}$ then we have

$$
\lim _{n \rightarrow \infty} \operatorname{Pr}\left\{X_{n}=\ell \mid X_{0}=0\right\}=\frac{a_{2}-a_{0}}{a_{2}}\left(\frac{a_{0}}{a_{2}}\right)^{\ell} \quad(\ell \geq 0) .
$$

that is, $X_{n}$ is positive recurrent. The distribution of $X_{n}$ converges to the stationary distribution.

(2) If $a_{0}=a_{2}$ then $X_{n}$ is null recurrenct and $X_{n} / \sqrt{2 a_{0} n}$ converges weakly to the absolute normal distribution. In particular, we have, as $n \rightarrow \infty$, and uniformly for all $\ell \leq C \sqrt{n}$ (where $C>0$ is an arbitrary constant)

$$
\operatorname{Pr}\left\{X_{n}=\ell\right\}=\frac{1}{\sqrt{n a_{0} \pi}} \exp \left(-\frac{\ell^{2}}{4 a_{0} n}\right)+\mathcal{O}\left(\frac{\ell}{n^{3 / 2}}\right) .
$$

(3) If $a_{0}>a_{2}$ then $X_{n}$ is non recurrent and

$$
\frac{X_{n}-\left(a_{0}-a_{2}\right) n}{\sqrt{\left(a_{0}+a_{2}-\left(a_{0}-a_{2}\right)^{2}\right) n}}
$$

converges weakly to the standard normal distribution. We also have, as $n \rightarrow \infty$ and uniformly for all $\ell \geq 0$ with $\left|\ell-\left(a_{0}-a_{2}\right) n\right| \leq C \sqrt{n}$ (where 


$$
\begin{aligned}
& C>0 \text { is an aribtrary constant) } \\
\operatorname{Pr} & \left\{X_{n}=\ell\right\} \\
= & \frac{1}{\sqrt{2 \pi\left(a_{0}+a_{2}-\left(a_{0}-a_{2}\right)^{2}\right) n}} \exp \left(-\frac{\left(\ell-\left(a_{0}-a_{2}\right) n\right)^{2}}{2\left(c+d-(c-d)^{2}\right) n}\right)+\mathcal{O}\left(\frac{1}{n}\right) .
\end{aligned}
$$

Remark . With a little bit more effort it can be shown that in the case $a_{0}=a_{2}$ the normalized discrete processes

$$
\left(\frac{X_{\lfloor t n\rfloor}}{\sqrt{2 a_{0} n}}, t \geq 0\right)_{n \geq 1}
$$

converges weakly to a reflected Brownian motion as $n \rightarrow \infty$; and for $a_{0}<a_{2}$ the processes

$$
\left(\frac{X_{\lfloor t n\rfloor}-t\left(a_{0}-a_{2}\right) n}{\sqrt{\left(a_{0}+a_{2}-\left(a_{0}-a_{2}\right)^{2}\right) n}}, t \geq 0\right)_{n \geq 1}
$$

converges weakly to the standard Brownian motion.

We split the proof into several lemmas.

Lemma 11. Let $N(x)$ be given by (14). Then we explicitly have

$$
N(x)=\frac{1-a_{1} x-\sqrt{\left(1-a_{1} x\right)^{2}-4 a_{0} a_{2} x^{2}}}{2 a_{0} a_{2} x^{2}} .
$$

The radius of convergence $x_{0}$ is given by

$$
x_{0}=\frac{1}{a_{1}+2 \sqrt{a_{0} a_{2}}}=\frac{1}{1-\left(\sqrt{a}_{0}-\sqrt{a}_{2}\right)^{2}} .
$$

If $a_{1}>0$ then $x_{0}$ is also the only singularity on the circle of convergence $|x|=x_{0}$. Furthermore, $N(x)$ has a local expansion of the form

$$
\begin{aligned}
N(x) & =\frac{a_{1}+2 \sqrt{a_{0} a_{2}}}{\sqrt{a_{0} a_{2}}}-\left(\frac{a_{1}+2 \sqrt{a_{0} a_{2}}}{\sqrt{a_{0} a_{2}}}\right)^{3 / 2} \cdot \sqrt{1-\left(a_{1}+2 \sqrt{a_{0} a_{2}}\right) x} \\
& +\mathcal{O}\left(1-\left(a_{1}+2 \sqrt{a_{0} a_{2}}\right) x\right)
\end{aligned}
$$

around its singularity $x=x_{0}$.

Proof. The proof is immediate.

Lemma 12. Suppose that $a_{0}<a_{2}$. Then the radius of convergence of $M_{0, \ell}(x)$ $(\ell \geq 0)$ is $x_{1}=1$, that is also a polar singularity of order 1 . Furthermore, we have

$$
\lim _{n \rightarrow \infty} \operatorname{Pr}\left\{X_{n}=K_{\ell}\right\}=\frac{a_{2}-a_{0}}{a_{2}}\left(\frac{a_{0}}{a_{2}}\right)^{\ell} \quad(\ell \geq 0) .
$$

Proof. First note that (for $\left.a_{0}<a_{2}\right)$ we have $N(1)=1 / a_{2}$ and $N^{\prime}(1)=\left(1-a_{2}+\right.$ $\left.a_{0}\right) /\left(a_{2}\left(a_{2}-a_{0}\right)\right)$. Thus,

$$
1-b x-a_{0} a_{2} z^{2} N(x)=\frac{a_{2}}{a_{2}-a_{0}}(1-x)+\mathcal{O}\left((1-x)^{2}\right) .
$$

and consequently

$$
M_{0, \ell}(x)=\frac{a_{2}-a_{0}}{a_{2}}\left(\frac{a_{0}}{a_{2}}\right)^{\ell} \frac{1}{1-x}+T(x)
$$


for $|x|<1 /\left(a_{1}+2 \sqrt{a_{0} a_{2}}\right)$, where $T(x)$ is an analytic function that has radius of convergence larger than 1 . (Note that $1 /\left(a_{1}+2 \sqrt{a_{0} a_{2}}\right)>1$.) Of course, this directly implies (19), compare also with Lemma 3.

The most interesting case is the case $a_{0}=a_{2}$.

Lemma 13. Suppose that $a_{0}=a_{2}$. Then the radius of convergence of $M_{\ell}(x)(\ell \geq 0)$ is $x_{1}=1$ that is also an algebraic singularity. Here we get, as $n \rightarrow \infty$, and uniformly for all $\ell \leq C \sqrt{n}$ (where $C>0$ is an arbitrary constant)

$$
\operatorname{Pr}\left\{X_{n}=\ell\right\}=\frac{1}{\sqrt{n a_{0} \pi}} \exp \left(-\frac{\ell^{2}}{4 a_{0} n}\right)+\mathcal{O}\left(\frac{\ell}{n^{3 / 2}}\right) .
$$

Proof. The essential difference between the present case and that of Lemma 12 is that $N(x)$ is not regular at $x=1$. We have to use the singular expansion (18) of Lemma 11 and obtain (around $x=1$ )

$$
1-b x-a_{0} a_{2} x^{2} N(x)=\sqrt{a_{0}} \sqrt{1-x}+\mathcal{O}(|1-x|) .
$$

Furthermore

$$
a_{0} x N(x)=1-\frac{1}{\sqrt{a_{0}}} \sqrt{1-x}+\mathcal{O}(|1-x|) .
$$

Thus, we can directly apply Lemma 6 and obtain (20).

The analysis of the final case $a_{0}>a_{2}$ is a little bit different from the previous ones. In the first two cases the singular behaviour of $M_{0, \ell}(x)$ around the point $x_{0}=1$ has governed the asymptotic behaviour of the coefficients. In the third case we will again work around the critial point $x_{0}=1$ but now with help of a saddle point method. The radius of convergence is larger than 1 .

Lemma 14. Suppose that $a_{0}>a_{2}$. Then $X_{n}$ satisfies a central limit theorem with mean value $\mathbf{E} X_{n} \sim\left(a_{0}-a_{2}\right) n$ and $\operatorname{Var} X_{n} \sim\left(a_{0}+a_{2}-\left(a_{0}-a_{2}\right)^{2}\right) n$. In particular we have the following local limit theorem:

$$
\begin{aligned}
& \operatorname{Pr}\left\{X_{n}=\ell\right\} \\
& \quad=\frac{1}{\sqrt{2 \pi\left(a_{0}+a_{2}-\left(a_{0}-a_{2}\right)^{2}\right) n}} \exp \left(-\frac{\left(\ell-\left(a_{0}-a_{2}\right) n\right)^{2}}{2\left(a_{0}+a_{2}-\left(a_{0}-a_{2}\right)^{2}\right) n}\right)+\mathcal{O}\left(\frac{1}{n}\right) .
\end{aligned}
$$

as $n \rightarrow \infty$ and uniformly for all $\ell \geq 0$ with $\left|\ell-\left(a_{0}-a_{2}\right) n\right| \leq C \sqrt{n}$ (where $C>0$ is an aribtrary constant)

Proof. Note first that $M_{0, \ell}(x)=M_{0,0}(x)\left(a_{0} x N(x)\right)^{\ell}$ and that $x_{1}=1$ is a regular point of $M_{0,0}(x)$ and $N(x)$. Thus, we can apply Lemma 8 for $r=1$ with $\rho(x)=$ $M_{0,0}(x)$ and $y(x)=a_{0} x N(x)$. Since $N(1)=1 / a_{0}$ and $N^{\prime}(1)=\left(1-a_{0}+a_{2}\right) /\left(a_{0}\left(a_{0}-\right.\right.$ $\left.a_{2}\right)$ ) we have $\mu(1)=1 /\left(a_{0}-a_{2}\right)$. Similarly we get $\sigma^{2}(1)=\left(a_{0}+a_{2}-\left(a_{0}-a_{2}\right)^{2}\right) /\left(a_{0}-\right.$ $\left.a_{2}\right)$. This completes the proof of Lemma 14 .

4.2. General Homogeneous Discrete QBD's. The next step is to generalize Theorem 1 to $m$-dimensional discrete QBD's. In the formulation of the theorem we will also use the interpretation of $X_{n}$ as a random walk on the "one-sided periodic graph" $G$. Recall that the vertices $V(G)$ of $G$ consist of infinite copies $V\left(K_{0}\right), V\left(K_{1}\right), \ldots$ of the vertices of the complete di-graph $K$ of $m$ nodes. For $v \in$ $V\left(K_{\ell}\right)$ then $\tilde{v}$ will denote the corresponding node in $K$. 
Theorem 2. Let $\mathbf{A}_{0}, \mathbf{A}_{1}, \mathbf{A}_{2}$ and $\mathbf{B}$ be square matrices of order $m$ with nonnegative elements with such that $\left(\mathbf{B}+\mathbf{A}_{0}\right) \mathbf{1}=\mathbf{1}$ and $\left(\mathbf{A}_{0}+\mathbf{A}_{1}+\mathbf{A}_{2}\right) \mathbf{1}=\mathbf{1}$, that is, the infinite matrix $\mathbf{P}$ from (10) is a transition matrix of a Markov process. Furthermore suppose that the matrices $\mathbf{B}$ is primitive irreducible, that no row of $\mathbf{A}_{0}$ is zero, and that $\mathbf{A}_{2}$ is non-zero.

Let $X_{n}$ denote a corresponding $Q B D$ with $X_{0} \in K_{0}$ (resp. a random walk on $G$ with $X_{0} \in K_{0}$ ) and let $x_{0}$ denote the radius of convergence of the entries of $\mathbf{N}(x)$ and $x_{1}$ that of $\mathbf{M}_{0,0}(x)$.

(1) If $x_{0}>1$ and $x_{1}=1$ then $X_{n}$ is positive recurrent and for all $v \in V(G)=$ $V\left(K_{0}\right) \cup V\left(K_{1}\right) \cup \cdots$ we have

$$
\lim _{n \rightarrow \infty} \operatorname{Pr}\left\{X_{n}=v\right\}=p_{v}
$$

where $\left(p_{v}\right)_{v \in V(G)}$ is the (unique) stationary distribution on $G$. Furthermore, the matrix $\mathbf{R}=\mathbf{A}_{0} \cdot \mathbf{N}(1)$ (where all eigenvalues have moduli $<1$ ) satisfies

$$
\mathbf{p}_{\ell+1}=\mathbf{p}_{\ell} \mathbf{R}
$$

in which $\mathbf{p}_{\ell}=\left(p_{v}\right)_{v \in K_{\ell}}$.

(2) If $x_{0}=x_{1}=1$ then $X_{n}$ is null recurrent and there exist $\rho_{v^{\prime}}>0\left(v^{\prime} \in V(K)\right)$ and $\eta>0$ such that, as $n \rightarrow \infty$, and (uniformly for all $\ell \leq C \sqrt{n}$ with an arbitrary constant $C>0$ )

$$
\operatorname{Pr}\left\{X_{n}=v\right\}=\rho_{\tilde{v}} \sqrt{\frac{1}{n \pi}} \exp \left(-\frac{\ell^{2}}{4 \eta n}\right)+\mathcal{O}\left(\frac{1}{n}\right) \quad\left(v \in V\left(K_{\ell}\right)\right) .
$$

(3) If $x_{1}>1$ then $X_{n}$ is non recurrent and there exist $\tau_{v^{\prime}}>0\left(v^{\prime} \in V(K)\right)$, $\mu>0$ and $\sigma>0$ such that, as $n \rightarrow \infty$ and uniform for all $\ell \geq 0$,

$$
\operatorname{Pr}\left\{X_{n}=v\right\}=\frac{\tau_{\tilde{v}}}{\sqrt{n}} \exp \left(-\frac{(\ell-\mu n)^{2}}{2 \sigma^{2} n}\right)+\mathcal{O}\left(\frac{1}{n}\right) \quad\left(v \in V\left(K_{\ell}\right)\right)
$$

Remark . Theorem 2 is, of course, a direct generalization of Theorem 1 . As above the second and the third case can be generalized to functional limit theorems in the following sense. For $v \in V\left(K_{\ell}\right)$ set $\hat{v}:=\ell$. Then $\hat{X}_{n}$ is a process on the non-negative integers and after a proper scaling $\hat{X}_{n}$ can be approximated by a reflected Brownian motion or by a Brownian motion. Note further that the matrix $\mathbf{R}=\mathbf{A}_{0} \cdot \mathbf{N}(1)$ in the first part of Theorem 2 is the classical $\mathbf{R}$-matrix for positive recurrent quasibirth-and-death processes, it also satisfies the equation $\mathbf{R}=\mathbf{A}_{0}+\mathbf{R B}+\mathbf{R}^{2} \mathbf{A}_{2}$, compare with [9, Theorem 6.2.1].

We also want to mention that that assumption that $\mathbf{B}$ is primitive irreducible is only used in the second part of the theorem.

Before we start with the proof of Theorem 2 we present a proper generalization of Lemma 11.

Lemma 15. Suppose that $\mathbf{B}$ is a primitive irreducible matrix and let $\mathbf{N}(x)=$ $\left(N_{v, v^{\prime}}(x)\right)_{v, v^{\prime} \in V(K)}$ denote the solution of (11). Then all functions $N_{v, v^{\prime}}(x)$ have a common radius of convergence $x_{0} \geq 1$. Furthermore, $x_{0}$ is the only singularity on the circle of convergence $|x|=x_{0}$ and there is a local expansion of the form

$$
\mathbf{N}(x)=\tilde{\mathbf{N}}_{1}-\tilde{\mathbf{N}}_{2} \sqrt{1-\frac{x}{x_{0}}}+\mathcal{O}\left(1-\frac{x}{x_{0}}\right)
$$

around its singularity $x=x_{0}$, where $\tilde{\mathbf{N}}_{1}$ and $\tilde{\mathbf{N}}_{2}$ are matrices with positive elements. 
Proof. The relation (11) is a system of $\mathrm{m}^{2}$ algebraic equation for the functions $N_{v, v^{\prime}}(x)$ that can be written in the form $\mathbf{y}(x)=\mathbf{F}(x, \mathbf{y}(x))$, where $\mathbf{y}(x)$ is just the vector of functions $N_{v, v^{\prime}}(x)$ and $\mathbf{F}(x, \mathbf{y})$ is a proper (non-linear) polynomial vector function with non-negative coefficients. By assumption $\mathbf{B}$ is irreducible (and nonnegative). Thus, the so-called dependency graph of this system is strongly connected. Consequently, by Lemma 4 all (algebraic) functions $N_{v, v^{\prime}}(x)$ have the same finite radius of convergence a squareroot singularity at $x=x_{0}$ of the form (24), where all entries of $\tilde{\mathbf{N}}_{1}$ and $\tilde{\mathbf{N}}_{2}$ are positive.

The assumption that $\mathbf{B}$ is primitive implies that all (sufficiently large) coefficients of the power series $N_{v, v^{\prime}}(x)$ are positive. This property ensures that $x=x_{0}$ is the only singularity on the circle of convergence $|x|=x_{0}$ (compare with the proof of Lemma 4.

Finally, we surely have $x_{0} \geq 1$. For, if $x_{0}<1$ then the coefficients of $N_{v, v^{\prime}}(x)$ are unbounded. However, the coefficients of $N_{v, v^{\prime}}(x)$ are probabilities (compare also with (13)) and thus bounded. This completes the proof of the lemma.

Proof. (Theorem 2) First, let us consider the case $x_{0}>1$ and $x_{1}=1$. By assumption, $x=1$ is a regular point of $\mathbf{M}(x)$ and, thus, the function

$$
f(x)=\operatorname{det}\left(\mathbf{I}-x \mathbf{B}-x^{2} \mathbf{A}_{0} \mathbf{N}(x) \mathbf{A}_{2}\right)
$$

is regular at $x=1$ and satisfies $f(1)=0$. Equivalently, 1 is an eigenvalue of the matrix $\mathbf{B}+\mathbf{A}_{0} \mathbf{N}(1) \mathbf{A}_{2}$. Since the matrix $\mathbf{B}+\mathbf{A}_{0} \mathbf{N}(1) \mathbf{A}_{2}$ is primitive irreducible, 1 is a simple eigenvalue. Consequently $x=1$ is a simple zero of $f(x)$ (and there are no further zeros on the circe $|x|=1$ ). Hence all functions of the inverse matrix $\left(\mathbf{I}-x \mathbf{B}-x^{2} \mathbf{A}_{0} \mathbf{N}(x) \mathbf{A}_{2}\right)^{-1}$ have a simple pole at $x=1$ (and no other singularities on the circe $|x|=1$ ). Thus, it follows as in the proof of Lemma 12 that the limits

$$
\lim _{n \rightarrow \infty} \operatorname{Pr}\left\{X_{n}=w\right\}
$$

exist for $w \in V(L)$. Similarly we get the existence of the corresponding limits for $v \in K_{\ell}$ and (21) with $\mathbf{R}=\mathbf{A}_{0} \mathbf{N}(1)$. Since $\sum_{v \in V(G)} p_{v}=1$ the moduli of all eigenvalues of $\mathbf{R}$ have to be smaller than 1 .

Next, suppose that $x_{0}=x_{1}=1$. Now $\mathbf{N}(x)$ is singular at $x=1$ and behaves like (24). We also have $f(1)=0$ (with $f(x)$ from above) and by using the definition of the determinant it also follows that $f(x)$ has a squareroot singularity of the form

$$
f(x)=c \sqrt{1-x}+\mathcal{O}(|1-x|),
$$

where $c \neq 0$. (If we consider $s=\sqrt{1-x}$ as a new variable then it follows as in the first part of the proof that $f(x)=\tilde{f}(s)$ has a simple zero in $s$. Thus, $c \neq 0$.)

Next, consider the powers $\left(x \mathbf{A}_{0} \mathbf{N}(x)\right)^{\ell}$. By assumption $x \mathbf{A}_{0} \mathbf{N}(x)$ has just postive entries (for real $x$ with $0<x \leq 1$ ). Hence, there exists a unique positive eigenvalue $\lambda(x)$ of $x \mathbf{A}_{0} \mathbf{N}(x)$ such that the moduli of all other eigenvalues are smaller than $\lambda(x)$. By continuity this is also true in a neighborhood of the real axis. Thus,

$$
\left(x \mathbf{A}_{0} \mathbf{N}(x)\right)^{\ell}=\lambda(x)^{\ell} \mathbf{Q}+\mathcal{O}\left(\lambda(x)^{(1-\eta) \ell}\right)
$$

for some matrix $\mathbf{Q}$ and some $\eta>0$. Since $\mathbf{N}(x)$ has a squareroot singularity at $x=1$, the eigenvalue $\lambda(x)$ has the same property:

$$
\lambda(x)=c_{1}-c_{2} \sqrt{1-x}+\mathcal{O}(|1-x|) .
$$


Hence, we are in a similar situation as in Lemma 13 and (22) follows; with the only difference that an additional factor $c_{1}^{\ell}=\lambda(1)^{\ell}$ appears. However, if $c_{1}<1$ then the probabilities do not sum up to 1 but the sum is bounded by $\mathcal{O}(1 / \sqrt{n})$. On the other hand, if $c_{1}>1$ then the sum of the probabilities does not converge. This provides $c_{1}=1$ and completes the proof of the second part of Theorem 2 .

Finally, suppose that $x_{1}>1$. Then we also have $x_{0}>1$. Thus, if we consider $\mathbf{M}_{0, \ell}(x)$ in a neighborhood of $x=1$ then all components of $\mathbf{M}_{0, \ell}(x)$ behave (almost) as powers of $\lambda(x)$ (the largest eigenvalue of $x \mathbf{A}_{0} \mathbf{N}(x)$ ) that is now analytic at $x=1$. Thus, we can again use the (saddle point) methods of Lemma 8 and obtain (23), however, again with a factor $\lambda(1)^{\ell}$. As above it follows that $\lambda(1)=1$ and we are done. Note that $\mu=1 / \lambda^{\prime}(1)$.

4.3. One-Dimensional Continuous QBD's. As in the discrete case we first study the one-dimensional continuous case first. Note that the results are of completely the same structure.

Theorem 3. Suppose that $q_{0}$ and $q_{2}$ are positive numbers, $q_{1}=-q_{0}-q_{2}$ and $b_{0}=-q_{0}$; and let $X(t)$ be the continuous $Q B D$ on the non-negative integers with generator matrix

$$
\mathbf{P}=\left(\begin{array}{cccccc}
b_{0} & q_{0} & 0 & 0 & \ldots & \\
q_{2} & q_{1} & q_{0} & 0 & \ldots & \\
0 & q_{2} & q_{1} & q_{0} & 0 & \ldots \\
0 & 0 & q_{2} & q_{1} & q_{0} & \ldots \\
\vdots & & \ddots & \ddots & \ddots & \ddots
\end{array}\right)
$$

(1) If $q_{0}<q_{2}$ then we have

$$
\lim _{t \rightarrow \infty} \operatorname{Pr}\{X(t)=\ell \mid X(0)=0\}=\frac{q_{2}-q_{0}}{q_{2}}\left(\frac{q_{0}}{q_{2}}\right)^{\ell} \quad(\ell \geq 0) .
$$

that is, $X(t)$ is positive recurrent. The distribution of $X(t)$ converges to the stationary distribution.

(2) If $q_{0}=q_{2}$ then $X(t)$ is null recurrent and $X(t) / \sqrt{2 q_{0} t}$ converges weakly to the absolute normal distribution. In particular, we have, as $t \rightarrow \infty$, and uniformly for all $\ell \leq C \sqrt{t}$ (where $C>0$ is an arbitrary constant)

$$
\operatorname{Pr}\{X(t)=\ell\}=\frac{1}{\sqrt{t q_{0} \pi}} \exp \left(-\frac{t^{2}}{4 q_{0} t}\right)+\mathcal{O}\left(\frac{\ell}{t^{3 / 2}}\right) .
$$

(3) If $q_{0}>q_{2}$ then $X(t)$ is non recurrent and

$$
\frac{X(t)-\left(q_{0}-q_{2}\right) t}{\sqrt{\left(q_{0}+q_{2}\right)\left(q_{0}-q_{2}\right)^{-2} t}}
$$

converges weakly to the standard normal distribution. We also have, as $t \rightarrow \infty$ and uniformly for all $\ell \geq 0$ with $\left|\ell-\left(q_{0}-q_{2}\right) t\right| \leq C \sqrt{t}$ (where $C>0$ is an aribtrary constant)

$$
\begin{aligned}
& \operatorname{Pr}\{X(t)=\ell\} \\
& \quad=\frac{1}{\sqrt{2 \pi\left(q_{0}+q_{2}\right)\left(q_{0}-q_{2}\right)^{-2} t}} \exp \left(-\frac{\left(\ell-\left(q_{0}-q_{2}\right) t\right)^{2}}{2\left(q_{0}+q_{2}\right)\left(q_{0}-q_{2}\right)^{-2} t}\right)+\mathcal{O}\left(\frac{1}{t}\right) .
\end{aligned}
$$

The structure of the proof of Theorem 3 is also very similiar to the proof of Theorem 1. We start with an analogue to Lemma 11. 
Lemma 16. Let $N(x)$ be the power series solution of

$$
N(x)=1+q_{1} x N(x)+q_{0} q_{2} x^{2} N(x)^{2},
$$

where $q_{1}=-q_{0}-q_{2}$ and $q_{0}$ and $q_{2}$ are positive, and set $\hat{N}(s)=\frac{1}{s} N\left(\frac{1}{s}\right)$. Then we explicitly have

$$
\hat{N}(s)=\frac{s+\left(q_{0}+q_{2}\right)-\sqrt{s^{2}+2\left(q_{0}+q_{2}\right) s+\left(q_{0}-q_{2}\right)^{2}}}{2 q_{0} q_{2}} .
$$

$\hat{N}(s)$ is singular at $s_{1}=-\left(q_{0}+q_{2}\right)+2 \sqrt{q_{0} q_{2}} \leq 0$ and $s_{2}=-\left(q_{0}+q_{2}\right)-2 \sqrt{q_{0} q_{2}}<0$

Furthermore, $\hat{N}(s)$ has a local expansion around $s_{1}=-\left(q_{0}+q_{2}\right)+2 \sqrt{q_{0} q_{2}}$ of the form

$$
\begin{aligned}
\hat{N}(s) & =\frac{1}{\sqrt{q_{0} q_{2}}}-\frac{1}{\left(\sqrt{q_{0} q_{2}}\right)^{3 / 2}} \sqrt{s-\left(-q_{0}-q_{2}+2 \sqrt{q_{0} q_{2}}\right)} \\
& +\mathcal{O}\left(\left|s-\left(-q_{0}-q_{2}+2 \sqrt{q_{0} q_{2}}\right)\right|\right) .
\end{aligned}
$$

With help of $\hat{N}(s)$ we can express the Laplace transforms $\hat{M}_{0, \ell}(s)$ of the function $t \mapsto \operatorname{Pr}\{X(t)=\ell \mid X(0)=0\}$ by

$$
\hat{M}_{0, \ell}(s)=\frac{\left(q_{0} \hat{N}(s)\right)^{\ell}}{s+q_{0}-q_{0} q_{2} \hat{N}(s)} .
$$

Instead of using Cauchy's formula we will apply the inverse Laplace transform to get some asymptotic information on

$$
\operatorname{Pr}\{X(t)=\ell \mid X(0)=0\}=\frac{1}{2 \pi i} \int_{c-i \infty}^{c+i \infty} \hat{M}_{0, \ell}(s) e^{s t} d s .
$$

Lemma 17. Suppose that $q_{0}<q_{2}$. Then the Laplace transform $\hat{M}_{0, \ell}(s)(\ell \geq 0)$ converges for $\Re(s)>0$. Furthermore, $\hat{M}_{0, \ell}(s)$ has a polar singularity of order 1 with residue $\left(q_{2}-q_{0}\right) / q_{2}\left(q_{0} / q_{2}\right)^{\ell}$ at $s=0$ and $s \hat{M}_{0, \ell}(s)$ can be analytically continued to $\Re(s)>-\eta$ for some $\eta>0$. Consequently,

$$
\lim _{t \rightarrow \infty} \operatorname{Pr}\left\{X(t)=K_{\ell}\right\}=\frac{q_{2}-q_{0}}{q_{2}}\left(\frac{q_{0}}{q_{2}}\right)^{\ell} \quad(\ell \geq 0) .
$$

Proof. First note that $\hat{N}(s)$ is surely analytic for $\Re(s)>-q_{0}-q_{2}+2 \sqrt{q_{0} q_{0}}$ and, thus, regular for $s=0$. Further, (for $q_{0}<q_{2}$ ) we have $\hat{N}(0)=1 / q_{2}$ and $\hat{N}^{\prime}(0)=$ $-1 /\left(q_{0}\left(q_{0}-q_{2}\right)\right)$. Thus,

$$
s+q_{0}-q_{0} q_{2} \hat{N}(s)=\frac{q_{2}}{q_{0}-q_{2}} s+\mathcal{O}\left(s^{2}\right) .
$$

and consequently

$$
\hat{M}_{0, \ell}(s)=\frac{q_{2}-q_{0}}{q_{2}}\left(\frac{q_{0}}{q_{2}}\right)^{\ell} \frac{1}{s}+\hat{T}(s),
$$

where $\hat{T}(s)$ is analytic for $\Re(s)>-\eta$ for some $\eta>0$. Of course, this directly implies (26).

The case $q_{0}=q_{2}$ is (again) quite interesting. 
Lemma 18. Suppose that $q_{0}=q_{2}$. Then the Laplace transform $\hat{M}_{0, \ell}(s)(\ell \geq 0)$ converges for $\Re(s)>0$. There is an algebraic singularity at $s=0$ and an analytic continuation to a region of the form $\Re(s)>-\eta,|\arg (s)|<\pi$ (for some $\eta>0$ ). Furthermore, we have, as $t \rightarrow \infty$, and uniformly for all $\ell \leq C \sqrt{t}$ (where $C>0$ is an arbitrary constant)

$$
\operatorname{Pr}\{X(t)=\ell \mid X(0)=0\}=\frac{1}{\sqrt{t q_{0} \pi}} \exp \left(-\frac{\ell^{2}}{4 q_{0} t}\right)+\mathcal{O}\left(\frac{\ell}{t^{3 / 2}}\right) .
$$

Proof. For $q_{0}=q_{2}$ we first obtain

$$
s+q_{0}-q_{0} q_{2} \hat{N}(s)=\sqrt{q_{0}} \sqrt{s}+\mathcal{O}(|s|) .
$$

Furthermore

$$
q_{0} \hat{N}(s)=1-\frac{1}{\sqrt{q_{0}}} \sqrt{s}+\mathcal{O}(|s|) .
$$

In fact, we are now almost in the same situation as in Lemma 6. However, instead of Cauchy's formla we use the inverse Laplace transform

$$
\operatorname{Pr}\{X(t)=\ell \mid X(0)=0\}=\frac{1}{2 \pi i} \int_{\gamma} \hat{M}_{0, \ell}(s) e^{s t} d s,
$$

where $\gamma=\gamma_{1} \cup \gamma_{2} \cup \gamma_{3} \cup \gamma_{4}$ is given by

$$
\begin{aligned}
& \gamma_{1}=\left\{s=-\frac{z}{t}|| z \mid=1, \Re z \leq 0\right\} \\
& \gamma_{2}=\left\{s=-\frac{z}{t} \mid 0<\Re z \leq \log ^{2} t, \Im z=1\right\} \\
& \gamma_{3}=\overline{\gamma_{2}} \\
& \gamma_{4}=\left\{s \mid \Re(s)=-\left(\log ^{2} t\right) / t, \Im(s)>1 / t\right\} .
\end{aligned}
$$

The integral over $\gamma_{4}$ is (as usual) negligible. If we use the substitution $s=-z / t$ for the remaining integral (that is, $s \in \gamma_{1} \cup \gamma_{2} \cup \gamma_{3}$ corresponds to $z \in \gamma^{\prime}$ ) then this integral is asymptotically of the same structure as the integral in Lemma 6. Hence we directly obtain (27).

The analysis of the final case $q_{0}>q_{2}$ relies (again) on a saddle point approximation.

Lemma 19. Suppose that $q_{0}>q_{2}$. Then $X(t)$ satisfies a central limit theorem with mean value $\mathbf{E} X(t) \sim\left(q_{0}-q_{2}\right) t$ and $\operatorname{Var} X(t) \sim\left(q_{0}+q_{2}\right)\left(q_{0}-q_{2}\right)^{-2} t$. In particular we have the following local limit theorem:

$$
\begin{aligned}
& \operatorname{Pr}\{X(t)=\ell\} \\
& \quad=\frac{1}{\sqrt{2 \pi\left(q_{0}+q_{2}\right)\left(q_{0}-q_{2}\right)^{-2} t}} \exp \left(-\frac{\left(\ell-\left(q_{0}-q_{2}\right) t\right)^{2}}{2\left(q_{0}+q_{2}\right)\left(q_{0}-q_{2}\right)^{-2} t}\right)+\mathcal{O}\left(\frac{1}{t}\right) .
\end{aligned}
$$

as $t \rightarrow \infty$ and uniformly for all $\ell \geq 0$ with $\left|\ell-\left(q_{0}-q_{2}\right) t\right| \leq C \sqrt{t}$ (where $C>0$ is an aribtrary constant)

Proof. Here we have $\hat{M}_{0, \ell}(s)=\hat{M}_{0,0}(s)\left(q_{0} \hat{N}(s)\right)^{\ell}$, where $s=0$ is a regular point of $\hat{M}_{0,0}(s)$ and $\hat{N}(s)$. The inverse Laplace transfrom

$$
\operatorname{Pr}\{X(t)=\ell \mid X(0)=0\}=\frac{1}{2 \pi i} \int_{-i \infty}^{i \infty} \hat{M}_{0, \ell}(s) e^{s t} d s
$$


is now concentrated around $s=0$. In fact, a usual saddle point method (that is completely similar to that of Lemma 7 resp. Lemma 8) leads to the proposed result.

The crucial step is to observe that $s=0$ is the saddle point or the function

$$
s \mapsto e^{s t+\ell \log \left(q_{0} \hat{N}(s)\right)}=e^{\left(t-\ell /\left(q_{0}-q_{2}\right)\right) s-\frac{1}{2} \ell\left(q_{0}+q_{2}\right)\left(q_{0}-q_{2}\right)^{-3} s^{2}+\mathcal{O}\left(s^{3}\right)}
$$

if $\ell=\left(q_{0}-q_{2}\right) t$ (and is also a proper approximation of a saddle point if $\ell=$ $\left.\left(q_{0}-q_{2}\right) t+\mathcal{O}(\sqrt{t})\right)$.

4.4. General Homogeneous Continuous QBD's. Finally we generalize Theorem 3 to $m$-dimensional continuous QBD's. One crucial step in the proof of Theorem 4 is to provide an analogue to Lemma 16. Since the matrix $\mathbf{A}_{1}$ has positive and negative entries we cannot apply Lemma 4 directly. Nevertheless, it seems that an analogue to Lemma 16 is actually true. For example, in the one-dimensional case we could have applied Lemma 4 by replacing $x$ by $-x$.

Theorem 4. Let $\mathbf{A}_{0}, \mathbf{A}_{1}, \mathbf{A}_{2}$ and $\mathbf{B}$ be square matrices of order $m$ such that $\mathbf{A}_{0}$ and $\mathbf{A}_{2}$ are non-negative and the matrices $\mathbf{B}$ and $\mathbf{A}_{1}$ have non-negative off-diagonal elements whereas the diagonal elements are stricly negative so that the row sums are all equal to zero: $\left(\mathbf{B}+\mathbf{A}_{0}\right) \mathbf{1}=\mathbf{0}$ and $\left(\mathbf{A}_{0}+\mathbf{A}_{1}+\mathbf{A}_{2}\right) \mathbf{1}=\mathbf{0}$, that is, the infinite matrix $\mathbf{Q}$ from (15) is a generator matrix of a homogeneous continuous $Q B D$ process. Furthermore suppose that the matrices $\mathbf{B}$ is primitive irreducible, that no row of $\mathbf{A}_{0}$ is zero, that $\mathbf{A}_{2}$ is non-zero, and that the system of equations (11) has a solution of the form (24), that is, all entries of $\mathbf{N}(x)$ have the same radius of convergence and the dominant singularity is of squareroot type.

Let $X(t)$ denote a corresponding $Q B D$ process with $X(0) \in K_{0}$ and let $\sigma_{0}$ denote the abscissa of convergence of $\hat{\mathbf{N}}(s)$ and $\sigma_{1}$ that of $\hat{\mathbf{M}}_{0,0}(s)$.

(1) If $\sigma_{0}<0$ and $\sigma_{1}=0$ then $X(t)$ is positive recurrent and for all $v \in V(G)=$ $V\left(K_{0}\right) \cup V\left(K_{1}\right) \cup \cdots$ we have

$$
\lim _{t \rightarrow \infty} \operatorname{Pr}\{X(t)=v\}=p_{v}
$$

where $\left(p_{v}\right)_{v \in V(G)}$ is the (unique) stationary distribution on $G$. Furthermore, the matrix $\mathbf{R}=\mathbf{A}_{0} \cdot \hat{\mathbf{N}}(0)$ (where all eigenvalues have moduli $<1$ ) satisfies

$$
\mathbf{p}_{\ell+1}=\mathbf{p}_{\ell} \mathbf{R}
$$

in which $\mathbf{p}_{\ell}=\left(p_{v}\right)_{v \in K_{\ell}}$.

(2) If $\sigma_{0}=\sigma_{1}=0$ then $X(t)$ is null recurrent and there exist $\rho_{v^{\prime}}>0\left(v^{\prime} \in\right.$ $V(K)$ ) and $\eta>0$ such that, as $t \rightarrow \infty$, and (uniformly for all $\ell \leq C \sqrt{t}$ with an arbitrary constant $C>0$ )

$$
\operatorname{Pr}\{X(t)=v\}=\rho_{\tilde{v}} \sqrt{\frac{1}{t \pi}} \exp \left(-\frac{\ell^{2}}{4 \eta t}\right)+\mathcal{O}\left(\frac{1}{t}\right) \quad\left(v \in V\left(K_{\ell}\right)\right) .
$$

(3) If $\sigma_{1}>0$ then $X(t)$ is non recurrent and there exist $\tau_{v^{\prime}}>0\left(v^{\prime} \in V(K)\right)$, $\mu>0$ and $\sigma>0$ such that, as $t \rightarrow \infty$ and uniform for all $\ell \geq 0$,

$$
\operatorname{Pr}\{X(t)=v\}=\frac{\tau_{\tilde{v}}}{\sqrt{t}} \exp \left(-\frac{(\ell-\mu t)^{2}}{2 \sigma^{2} t}\right)+\mathcal{O}\left(\frac{1}{t}\right) \quad\left(v \in V\left(K_{\ell}\right)\right) .
$$

Proof. The proof is a direct extension of the proof of Theorem 3 by using the ideas of the proof of Theorem 2 . 


\section{REFERENCES}

[1] E. A. Bender, Asymptotic methods in enumeration, SIAM Rev. 16 (1974), 485-515.

[2] M. Drmota, Asymptotic distributions and a multivariate Darboux method in enumeration problems, J. Combinat. Theory, Ser. A 67 (1994), 169-184.

[3] M. Drmota, A bivariate asymptotic expansion of coefficients of powers of generating functions, Europ. J. Combinatorics 15 (1994), 139-152.

[4] M. Drmota, Systems of functional equations, Random Struct. Algorithms 10 (1997), 103-124.

[5] M. Drmota, Discrete random walk on one-sided "periodic graphs", Discr. Math. Theor. Comput. Sci. AC (Discrete Random Walks) (2003), 83-94.

[6] P. Flajolet and A. M. Odlyzko, Singularity analysis of generating functions, SIAM J. Discrete Math. 3 (1990), 216-240.

[7] L. Kaup and B. Kaup, Holomorphic Functions of Several Variables, Studies in Mathematic, de Gruyter, Berlin, 1983,

[8] W. Kuich and F. J. Urbanek, Infinite linear systems and one-counter languages, Theoret. Comput. Sci., 22 (1983), 95-126.

[9] G. Latouche and V. Ramaswami, Introduction to matrix analytic methods in stochastic modeling, ASA-SIAM Series on Statistics and Applied Probability, Society for Industrial and Applied Mathematics (SIAM), Philadelphia, PA, 1999.

[10] M. F. Neuts, Matrix-geometric solutions in stochastic models, Johns Hopkins Series in the Mathematical Sciences, 2, Johns Hopkins University Press, Baltimore, Md., 1981.

[11] M. F. Neuts, Structured stochastic matrices of $M / G / 1$ type and their applications, Probability: Pure and Applied, 5, Marcel Dekker Inc., New York, 1989.

[12] A. M. Odlyzko, Asymptotic enumeration methods, In: Handbook of combinatorics, Vol. 1, 2, 1063-1229, Elsevier, Amsterdam, 1995.

[13] H. Prodinger, Further results on a problem of Knödel concerning the analysis of bin-packing, in: Number-theoretic analysis (Vienna, 1988-89), Lecture Notes in Math., 1452, 193-198, Springer, Berlin, 1990.

[14] R. Sedgewick and P. Flajolet, An Introduction to the Analysis of Algorithms, Addison Wesley, 1996.

[15] J. S. Vitter and P. Flajolet, Average-Case Analysis of Algorithms and Data Structures, in Handbook of Theoretical Computer Science, J. van Leeuwen, Ed., vol. A: Algorithms and Complexity. North Holland, 1990, ch. 9, pp. 431-524. 